ARTICLE

\title{
Engineering stable radicals using photochromic triggers
}

Xuanying Chen ${ }^{1,2}$, Wandong Zhao ${ }^{2}$, Gleb Baryshnikov (1) ${ }^{3}$, Michael L. Steigerwald ${ }^{4}$, Jian Gu', Yunyun Zhou', Hans Ågren ${ }^{3}$, Qi Zou (1) ${ }^{2,4 凶}$, Wenbo Chen ${ }^{2 凶}$ \& Liangliang Zhu (iD) ${ }^{凶}$

Long-standing radical species have raised noteworthy concerns in organic functional chemistry and materials. However, there remains a substantial challenge to produce long-standing radicals by light, because of the structural dilemmas between photoproduction and stabilization. Herein, we present a pyrrole and chloride assisted photochromic structure to address this issue. In this well-selected system, production and stabilization of a radical species were simultaneously found accompanied by a photochemical process in chloroform. Theoretical study and mechanism construction indicate that the designed $\pi$-system provides a superior spin-delocalization effect and a large steric effect, mostly avoiding possible consumptions and making the radical stable for hours even under an oxygen-saturated condition. Moreover, this radical system can be applied for a visualized and quantitative detection towards peroxides, such as 2,2,6,6-tetramethylpiperidine-1-oxyl, hydrogen peroxide, and ozone. As the detection relies on a radical capturing mechanism, a higher sensing rate was achieved compared to traditional redox techniques for peroxide detection.

\footnotetext{
${ }^{1}$ State Key Laboratory of Molecular Engineering of Polymers, Department of Macromolecular Science, Fudan University, Shanghai 200438, China. ${ }^{2}$ Shanghai Key Laboratory of Materials Protection and Advanced Materials in Electric Power, Shanghai University of Electric Power, Shanghai 200090 China. ${ }^{3}$ Division of Theoretical Chemistry and Biology, School of Chemistry, Biotechnology and Health, KTH Royal Institute of Technology, SE-10691 Stockholm, Sweden. ${ }^{4}$ Department of Chemistry, Columbia University, New York, NY 10027, USA. ${ }^{{ }^{2}}$ email: qzou@shiep.edu.cn; wenbochen@shiep.edu.cn; zhuliangliang@fudan.edu.cn
} 
R adicals that can be stabilized in chemical systems have played an important role in regulating chemical reactivity and material physical properties ${ }^{1-3}$. By delocalizing spin and blocking subsequent reactions, chemists have developed several molecular structures (e.g. multi-cyano skeletons ${ }^{4}$, tetramethyl azacyclic systems ${ }^{5}$, polythiophenes ${ }^{6,7}$, azulenes ${ }^{8}$, porphyrins $^{9}$, and other extended aromatic compounds ${ }^{10}$ ) for achieving stable radical species. These systems also show remarkable potential in organic optoelectronics, magnetic materials and magnetic resonance imaging, non-linear optical devices, as well as energy storage ${ }^{11,12}$. Nevertheless, the stable radical generations thus far have largely relied on redox reactions or thermal processes, lacking the convenience from the perspective of material manipulation. In contrast, light stimulus is typically precise and rapid, and can provide contactless spatial and temporal control ${ }^{13-17}$. Although it is popular to employ photon (or photothermal effect) to generate intermediate radicals for reactions, to straightforwardly produce stable radical species by light, which will be in favor of further developing photochemical methodology as well as for steady-state material application, is still challenging but desirable.

To produce stable radicals by light, we need to overcome the dilemmas in chemical design between the photoproduction and the stabilization factors. There are two facts that mostly hamper the above-mentioned hypothesis: (1) unpaired electrons produced upon photoirradiation can easily suffer from ultrafast back pairing relaxation; (2) there is lack of structural characteristics to stabilize radicals in chemical systems even if they are formed. Here we note that the photochromism, as a representative photochemical behavior, which can undergo rapid and efficient photocontrol process accompanied by dramatic $\pi$-electron rearrangement to change the apparent $\operatorname{color}^{18-23}$, may have potential to fulfill the task. Photochromic materials have received widespread interest in probes, information storage, logic gates, selfassemblies, biological materials, etc ${ }^{24,25}$. During a photochromic process, the exciton can undergo an intermediary transition to an excited state. In this way, it exerts diverse conversion possibilities and can ideally ensure a follow-up coupling with orbitals of nearby molecules. Photochromism can also coexist with radical species in some presupposed magnetic molecular structures, featuring a superior orthogonality in material design ${ }^{26-29}$. On the other hand, relatively stable radicals formed from hexaarylbiimidazole for photochromism were also reported ${ }^{30-32}$.
However, to produce even stronger and more stable radical properties for steady-state material application triggered by photochromic process remains difficult, simply because the existed photochromic skeletons lack a sufficient spindelocalization effect to stabilize the radicals once formed.

Dithienylethene is a typical photochromic moiety enabling a relatively large and planar molecular geometry during photocyclization ${ }^{33,34}$. In another hand, pyrrole and chloride can work as effective assisted groups to stabilize the radicals in large $\pi$-conjugated systems ${ }^{35,36}$. We envisage that pyrrole and chloride assisted conjugated structures based on dithienylethenes can provide a superior chemical environment for radical growth by light and radical stabilization by delocalizing spin. Our inspiration origins not only from previous reports on the photochemical feature of dithienylethenes, but also from the fact that the intrinsic properties of dithienylethenes can readily be modulated by rational backbone modification. Therefore, by clamping two chlorinated dithienylethenes into a modified pyrrole center, a sufficiently large conjugated system with photochromic triggers is designed and synthesized (compound 1, see Fig. 1a). To get deeper insight into the intrinsic structural rationality of compound 1 , we also extend to more systems by synthesizing a series of reference compounds, namely 2, 3, and 4, for control study (Fig. 1a), from the perspective of regulating the chlorinated dithienylethene and pyrrole. And we eventually find that the compound 1 can perform as we expected by optimization (Fig. 1b).

\section{Results}

Radical properties upon irradiation. UV-vis spectra of compound 1 changed significantly within 1 min upon irradiation with $365 \mathrm{~nm}$ light (Fig. 2a), suggesting a high sensitivity and fast response to UV irradiation. A typical absorption band at $\sim 500 \mathrm{~nm}$ emerged, indicating a typical photochromic behavior accompanied by the photocyclization of dithienylethene from its ringopen form to ring-closed one. This is further verified by fluorescence quenching (Fig. 2b).

To our surprise, however, an additional absorption band at $\sim 630 \mathrm{~nm}$ also appeared upon a continuous irradiation in chloroform, and continued to grow with the prolongation of the irradiation time till $\sim 5 \mathrm{~min}$ (Fig. 2a). To further investigate the unusual dual band characteristic, UV-vis spectra of compound
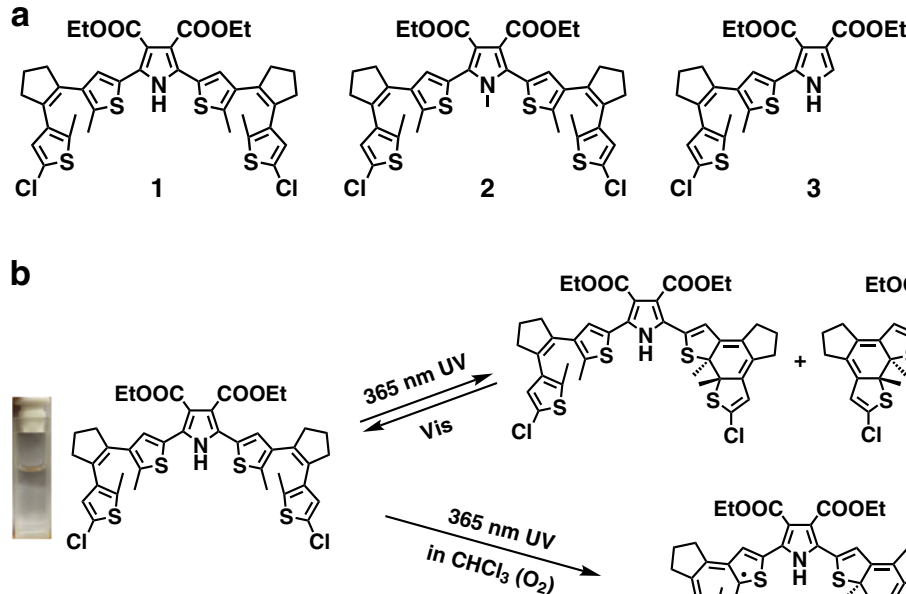

3
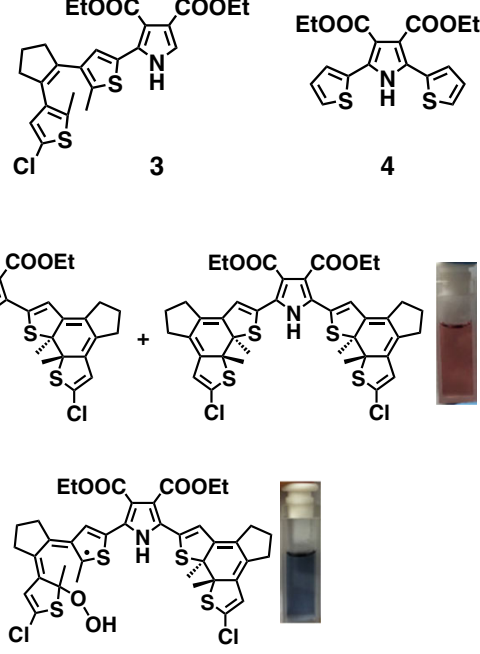

Fig. 1 Research outline. a Illustration for related chemical structures: compounds 1-4. b An outcome of photochromic switching and radical production processes of compound 1 controlled by light in oxygen-saturated chloroform. 

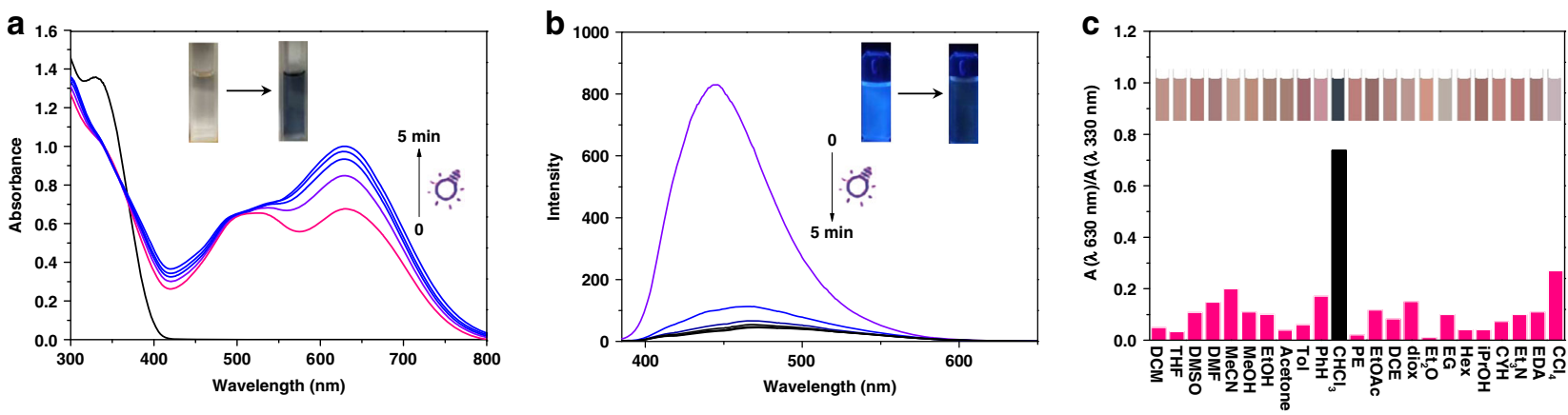

Fig. 2 Photochemical properties. a UV-vis absorption spectral and $\mathbf{b}$ fluorescence spectral changes of compound 1 ( $5.0 \times 10^{-5} \mathrm{M}$ ) in oxygen-saturated $\mathrm{CHCl}_{3}$ solution upon irradiation with $365 \mathrm{~nm}$ light at room temperature. $\mathbf{c}$ The absorption ratio $\left(A_{630 \mathrm{~nm}} / A_{330} \mathrm{~nm}\right)$ of compound 1 in a series of solvents and the corresponding color changes (inset).

1 were measured in a series of different solvents (the details including the solvent abbreviations are listed in Supplementary Fig. 1). It was found that only one band centered at around 500 $\mathrm{nm}$ emerged after UV irradiation with a colorless solution converted to pink, except in chloroform. High performance liquid chromatography (HPLC) showed that two new peaks formed simultaneously upon the photoirradiation (Supplementary Fig. 2), suggesting that both dithienylethene units of compound 1 participate in the chemical process with different efficiency in these solvents except chloroform. To exclude the influence of residue acid in chloroform on the corresponding properties of compound 1, we first determine the purity of chloroform solvents by gas chromatography (GC), suggesting the purity of the solvents is quite high without any impurity (Supplementary Figs. 3 and 4). Then UV-vis spectra were measured in different alkali-treated and acid-treated chloroform under the same conditions, respectively. The absorption changes in these treated chloroform solvents show the same tendency as used in the common chloroform (Supplementary Figs. 5-7). Thus, we conclude that the gradient color from pink to blue in chloroform should be attributed to an additional mechanism, rather than a common photochromism.

A radical formation upon photochemical triggering can then be inferred, since a radical can easily result in a significant absorption shift ${ }^{1-3,11,12}$. The absorbance ratio $\left(\mathrm{A}_{630} \mathrm{~nm} / \mathrm{A}_{330 \mathrm{~nm}}\right.$, Fig. 2c) reflects the relative yield of the possible radical species, suggesting that such a process in chloroform is outstandingly efficient. As traditional photoswitching compounds, the cyclization and cycloreversion processes of the dithienylethene can be well monitored by ${ }^{1} \mathrm{H}$ NMR spectra ${ }^{37,38}$. In our case, compound 1 in DMSO- $d_{6}$ connects to a standard photocyclization characterization with obvious ${ }^{1} \mathrm{H}$ NMR shifts (Fig. 3 and Supplementary Fig. 9), similar to the typical photochromism of dithienylethenes ${ }^{33,34}$. In contrast, the ${ }^{1} \mathrm{H}$ NMR spectra of compound 1 in $\mathrm{CDCl}_{3}$ only show tiny signal change before and after UV irradiation (see Fig. 3). Besides, we see from Supplementary Fig. 8 that a small chemical shift change on proton and the linewidth is slightly broadened upon irradiation of compound 1 in $\mathrm{CDCl}_{3}$. We reason that the lack of a huge chemical shift change and the line broadening is due to the localization of a possible radical on the carbon, hence it has minimal contact effect on the proton in the nearby groups. Due to the short electron relaxation time as well as the fast rotation of these groups, the persudo-contact effect in the hyperfine interaction can be decoupled and shows little effect on the proton spectrum $^{39,40}$. These results strengthened the fact that a radical process may occur within chloroform, which revealed a relatively lower sensitivity in ${ }^{1} \mathrm{H}$ NMR change but competitively weakened the ratio of the photocyclization (see a tiny ${ }^{1} \mathrm{H}$ NMR signal of protons $\mathrm{Ha}^{\prime}$ and $\mathrm{Hb}^{\prime}$ in Fig. $3 \mathrm{~b}$ ).
Electron paramagnetic resonance (EPR) measurements provided direct evidence of the radical formation and suggested that radicals with a magnetic field strength at $3200-3400 \mathrm{mT}$ were generated in $\mathrm{CDCl}_{3}$ solution after UV irradiation (Fig. 3b). In contrast, the EPR spectra did not show radical signals in DMSO$d_{6}$ solution after UV irradiation (Fig. $3 c, d$ ). These results feature a solvent-dependence of yielding the radical. It is worth noting that such a radical could be easily detected by EPR even without any radical trapping agent, suggesting that they are quite stable. The lifetime of the radicals is further determined to be hours by a decay curve based on the absorbance at $630 \mathrm{~nm}$ in chloroform under an oxygen-saturated condition (Fig. 3e, f).

Proposed radical production mechanism. The reference compounds 2, 3, and 4 were synthesized to get insight into the mechanism of the radical production. The UV-vis spectra of the reference compounds 2, 3 and 4 are shown in Fig. $4 c-e$, and here we can only observe the photochemical process on compounds 2 and 3 rather than compound 4 , signifying the importance of the existence of the dithienylethene unit. That means the "photochromic triggers" are necessary. Similarly, ${ }^{1} \mathrm{H}$ NMR peaks of compounds 2 and 3 in $\mathrm{CDCl}_{3}$ did not change remarkably upon irradiation with $365 \mathrm{~nm}$ light (Supplementary Figs. 10 and 11). The EPR spectra of compounds 2 and 3 also indicate that radicals exist in $\mathrm{CDCl}_{3}$ with $365 \mathrm{~nm}$ irradiation (Supplementary Fig. 12). However, the absorbance ratio $\left(\mathrm{A}_{630 \mathrm{~nm}} / \mathrm{A}_{330 \mathrm{~nm}}\right)$ of compounds 2 and 3 is relatively low, suggesting a less efficient production or existence of radicals in compounds 2 and 3 than in compound 1 . Therefore, the big structural regulation in compound 1 is definitely helpful for the production and stabilization of the phototriggered radical species. We can even see that there is no EPR signal when the blank chloroform solvent was illuminated under the same conditions (Supplementary Fig. 12), suggesting that the radical signal indeed originates from the studied structures rather than from possible free radical residue in the solvents or other disruptors.

According to a relevant report ${ }^{41}$, the EPR signal position near the magnetic field strength of 3350 indicates that there exist oxygen species in the system. The blue solution cannot be obtained for compound 1 in degassed chloroform upon UV irradiation (Fig. 4a), further proving that the radical formation in our case requires the participation of oxygen. Such a control study indicates that for further ensuring the stabilization of the possibly yielded radicals, a dehydrogenation effect by oxygen coupling was involved for the aid of our experiments. On the other hand, a solution of compound 1 with 10 equiv. of thiophenol in benzene could also turn to blue with UV irradiation (Fig. 4b), similar to the same absorption change with that in oxygen-saturated chloroform, featuring a dehydrogenation 
a

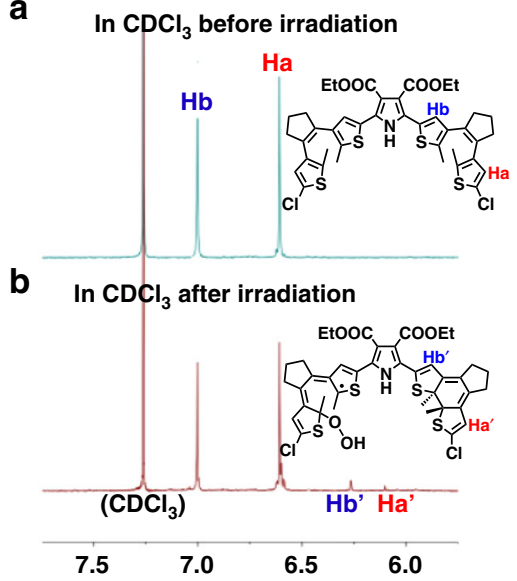

C

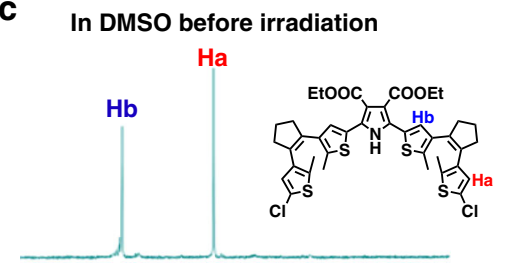

d In DMSO after irradiation

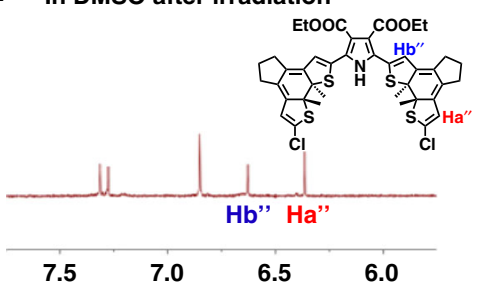

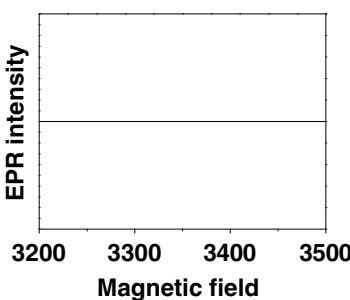
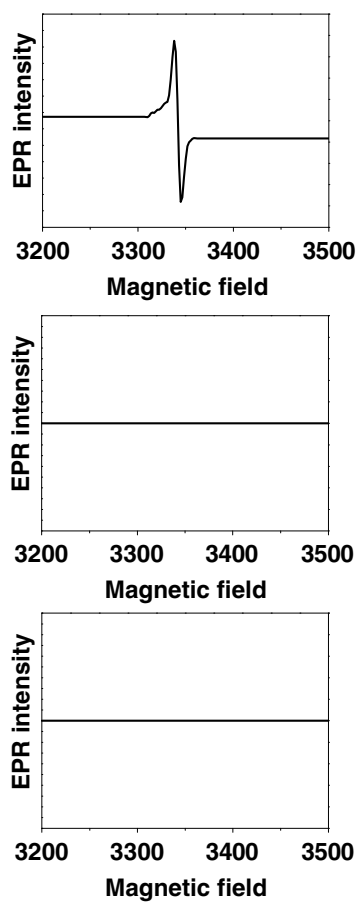

e

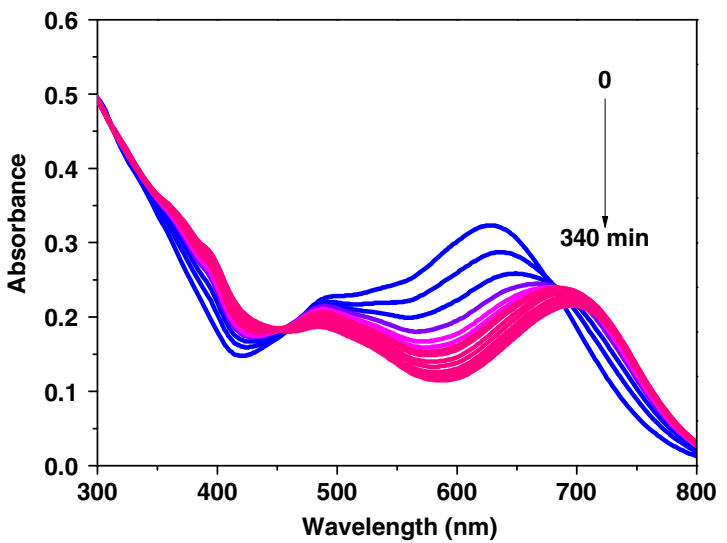

f

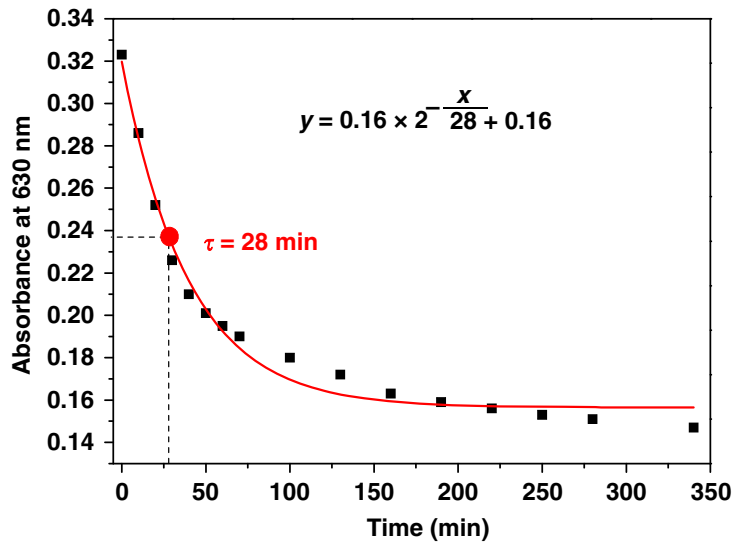

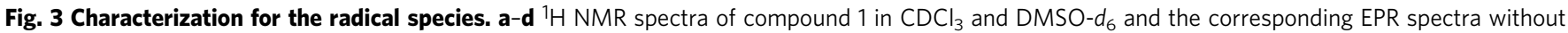
and with irradiation of $365 \mathrm{~nm}$ light at $25^{\circ} \mathrm{C}$, respectively. e UV-vis absorption spectral changes and f the decay curve of compound 1 ( $1.0 \times 10^{-5} \mathrm{M}$ ) in oxygen-saturated $\mathrm{CHCl}_{3}$ solution after withdrawing the photoirradiation.

effect on the peroxide-based radical formation in the presence of an activable hydrogen donor. And thus, we can propose that the excited state $1^{\star}$ (which can also be referenced from a typical photochromic process, see Fig. 4f) as a diradical firstly forms upon photoexcitation with UV light. Followed by this photochromic trigger, in the presence of $\mathrm{O}_{2}, 1^{\star}$ can be favorable to combine with oxygen to give an intermediate. The intermediate can then immediately extract a relatively active hydrogen atom (for instance, $\mathrm{H}-\mathrm{CCl}_{3}$ and $\mathrm{H}-\mathrm{SC}_{6} \mathrm{H}_{5}$ in our case) to form a peroxide-based monoradical species $1^{\prime}$, corresponding to the blue color of the solution which is much more stable. While compound 1 exhibited an identical signal position in EPR and absorption spectra with that of compound 3, we can conclude that the radical species is a monohydroperoxide one (like the structure showed in Fig. 4f), leaving the other dithienylethene still photocyclizable (see the tiny ${ }^{1} \mathrm{H}$ NMR signal shifted in Fig. 3 and Supplementary Fig. 8 for details, respectively).

We believe that the above-mentioned mechanism is reasonable because $\mathrm{CHCl}_{3}$ is the relatively most effective hydrogen donor upon irradiation ${ }^{42,43}$. In this way, this process can be perfectly demonstrated by the presence of an even strong hydrogen donor (thiophenol, see Fig. 4b). The radical species is still difficult to be directly detected by mass spectrometry simply because of its oxygen and $\mathrm{CHCl}_{3}$ dependence. Therefore, only unreacted molecular ion peaks can be found (see Supplementary Fig. 13 for comparison). However, an indirect approach can be employed by the addition of 2,2,6,6-tetramethylpiperidine-1-oxyl (TEMPO) to covalently couple with the monoradical species to prove this issue. Key fragment peaks (e.g. $m / z=730.1854$ ) upon the coupling structure were observed in Fig. 4i (see also the illustration for the coupling and fragment analysis in Supplementary Fig. 14), the high-resolution value of which showed a very close agreement with that calculated (mass error $<9 \mathrm{ppm}$ ). Besides, preparative HPLC was employed to isolate the adduct of photo-irradiated compound 1 coupled with TEMPO (from the new compound signal with the retention time of $6.388 \mathrm{~min}$, see details showed in Fig. $4 \mathrm{~g}$ and Supplementary Fig. 15). As shown in the ${ }^{1} \mathrm{H}$ NMR spectrum (see Fig. 4h), several characteristic peaks were observed, including the pyrrole proton, thiophene protons and peroxide proton. These results for the adduct show good agreement with the mechanism we proposed. In addition, we emphasize that another possible mechanism regarding a participation of $\mathrm{Cl}$ radical can be ruled out, since a common photochromic phenomenon was also observed in carbon tetrachloride (see Fig. $2 \mathrm{c}$ and Supplementary Fig. 1v). These control studies further supported the suggested route as shown in Fig. $4 \mathrm{f}$.

As photochromism is always an incomplete photoconversion process, we are unfortunately unable to employ single crystal XRD to precisely characterize the radical structure. We instead carried out a calculation study to verify our assumption and to analyze the stability. As can be seen from the spin-density analysis (Supplementary Fig. 16 and Supplementary Table 1), the radical can be assigned as a $\pi$-type strongly delocalized one. This fact actually explains its high stability. We can see the prevailing 


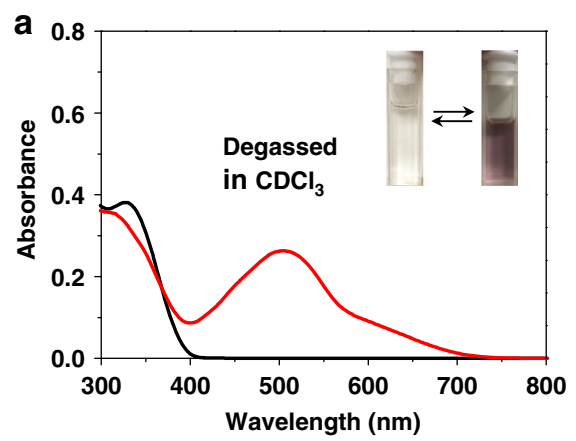

f
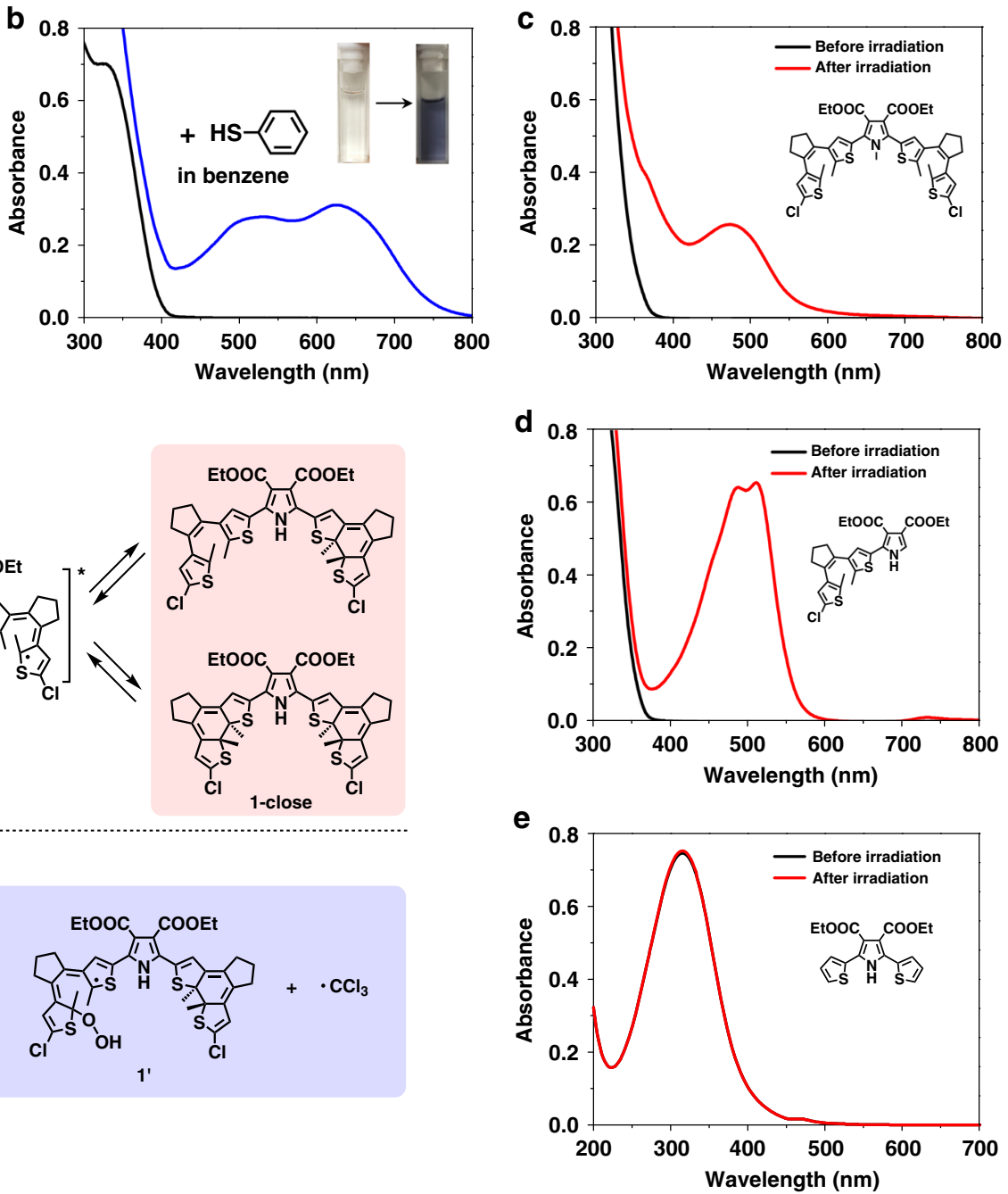

i

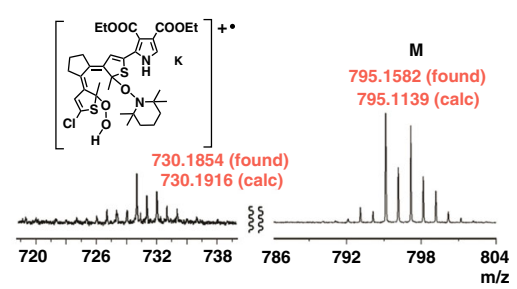

Fig. 4 Mechanism study on the radical species. a UV-vis absorption spectral changes of compound $1\left(1.0 \times 10^{-5} \mathrm{M}\right)$ upon irradiation with $365 \mathrm{~nm}$ light for 5 min in degassed $\mathrm{CHCl}_{3}$. Inset: the corresponding solution color change. $\mathbf{b}$ UV-vis absorption spectral changes of compound 1 (1.0 $\times 10^{-5} \mathrm{M}$ ) with 10 equiv. of thiophenol in benzene upon irradiation with $365 \mathrm{~nm}$ light for $5 \mathrm{~min}$ at $25^{\circ} \mathrm{C}$. Inset: the corresponding photographic images upon irradiation with $365 \mathrm{~nm}$ light for $5 \mathrm{~min}$. UV-vis absorption spectral changes of compounds 2 (c), 3 (d), 4 (e) in $\mathrm{CHCl}_{3}\left(1.0 \times 10^{-5} \mathrm{M}\right)$ upon irradiation with $365 \mathrm{~nm}$ light. $\mathbf{f} \mathrm{A}$ proposed radical generation process in molecule 1. $\mathbf{g} \mathrm{HPLC}$ spectra of compound 1 (above) and compound 1 after irradiation with TEMPO (below) at $25^{\circ} \mathrm{C}$ under following conditions: Agilent $\mathrm{C} 18$ column, acetonitrile-water $(6: 4, \mathrm{v} / \mathrm{v})$ as mobile phase, the detection wavelength of $254 \mathrm{~nm}$. $\mathbf{h}$ Partial ${ }^{1} \mathrm{H}$ NMR spectrum of isolated TEMPO-coupled compound corresponding to the new peak at retention time of 6.388 min in HPLC. i MS (MALDI-TOF) of photoirradiated compound 1 in oxygen-saturated $\mathrm{CHCl}_{3}$ solution with addition of 10 equiv. of TEMPO.

contribution to the spin-density plots from the S-containing dithienylethene branches and also from the central pyrrole ring, suggesting the necessity of an alternating structure. The singleoccupied molecular orbitals (SOMOs) are completely responsible for the observed spin-density patterns. Here the spin-density plots can be considered as the square of SOMO orbitals whereas the contribution from other alpha-spin orbitals is found to be negligible. Regarding the calculated isotropic g-factor, a considerable positive deviation of the average g-factor from that for the free electron can be observed: 2.004 vs. 2.002 . By analyzing the g-tensor components (Supplementary Table 1), we see that the XX and YY components for the radical are almost the same and vary in the range 2.004-2.006, while the $\mathrm{ZZ}$ component is much smaller (around 2.004). Such an anisotropy of the g-tensors is typical for stable $\pi$-type radicals ${ }^{4-46}$. In our case, the radical is placed in the $\mathrm{XY}$ plane, while $\mathrm{Z}$ axis is perpendicular to this plane. On the other hand, some theoretical calculations on ${ }^{1} \mathrm{H}$ NMR (Supplementary Table 2) and the absorption (Supplementary Fig. 17) of the proposed radical species were also performed and summarized in Supplementary Information. The calculations 
show good agreement in the signal positions with the experimental results for compound 1 and its photostationary state. The photostability of the photocyclized skeleton is good, as a relatively obvious photobleaching can only be observed after $24 \mathrm{~h}$ upon continuous irradiation (Supplementary Fig. 18).

Application for visualizing ozone detection. The UV-vis absorption spectra and color of the radical species of compound 1 are sensitive to peroxides, such as TEMPO and hydrogen peroxide. As shown in Supplementary Fig. 19, after adding TEMPO and hydrogen peroxide, respectively, UV-vis absorption spectra of compound 1 at the photostationary state in $\mathrm{CHCl}_{3}$ changed with the disappearance of the characteristic peak at $630 \mathrm{~nm}$, due to the radical capturing effect. Therefore, the photostationary state of compound 1 in $\mathrm{CHCl}_{3}$ can be used as a detector for peroxide with naked-eye recognition. To better monitor the qualitative sensing ability, we measured UV-vis absorption spectral changes of compound 1 at the photostationary state in $\mathrm{CHCl}_{3}$ in the presence of increasing concentrations of TEMPO and hydrogen peroxide, respectively, and plotted the corresponding absorbance at $630 \mathrm{~nm}$ with respect to the concentrations of TEMPO and hydrogen peroxide (Supplementary Figs. 20 and 21). It is found that the absorbance at $630 \mathrm{~nm}$ could be well fitted to the concentration of TEMPO and hydrogen peroxide, respectively, suggesting that compound 1, as a photochromic probe, could achieve the practical calibration and quantitative determination of peroxide.

Therefore, we employed this material to construct an easy-touse probe for the detection of ozone by colorimetry. Peroxides, particularly ozone, are important oxidants that have many industrial and consumer applications related to oxidation, whereas an excessive production of peroxides makes them potent respiratory hazards and pollutants near the ground level ${ }^{47,48}$. When ozone flow generated from the ozonator (see Supplementary Fig. 22) was immersed into the $\mathrm{CHCl}_{3}$ solution of compound 1 at the photostationary state, the blue solution converted to deep red within $20 \mathrm{~s}$ along with an absorption change (Fig. 5a). In this way, it can serve as a rapid detector for ozone with naked-eye visualization. As presented in Fig. 5b, the change rate of compound 1 with $\mathrm{O}_{3}$ was the fastest one among the various types of gas (see also the fitting process in detail in Supplementary Fig. 23), featuring that a possible radical capturing mechanism played key roles, not just an oxidation process. Besides, we prepared the gel 1 from compound 1 by blending the polyvinyl chloride with the corresponding chloroform solution as shown in Fig. 5c. The apparent color could turn to blue from colorless after UV irradiation similarly. Upon $\mathrm{O}_{3}$ bubbling, the blue gel quickly turned to pink gel (from 3 to 2). This suggests that the gel based on compound 1 at the photostationary state can work well as a portable $\mathrm{O}_{3}$ detector. We can calculate the $\mathrm{O}_{3}$ volume from the flow rate per unit time, thus to achieve a quantitative ozone detection by this material. Such a detection with a radical capturing mechanism ensures a higher sensing rate compared to traditional redox techniques for peroxide detection.

\section{Discussion}

In summary, a unique stable radical formation strategy triggered by photochromism has been demonstrated. The molecular design of a pyrrole and chloride assisted photochromic structure has been proved to play the key role. Our compound shows a two-stage response to UV light, due to the generation of an unusual radical other than the traditional photocyclization. It is proposed that the dehydrogenation effect helps to stabilize the electron departure upon photoexcitation to yield a peroxide-based species. Furthermore, the structural design for the conjugated structure provides a spin-delocalization effect and a large steric effect, making the radical stable under oxygen-saturated conditions for hours. It is argued that such a radical species can be manipulated as radical trapping agents to detect peroxides by colorimetry, including TEMPO, hydrogen peroxide, and ozone. These detections can be quantitative and portable. The presented photochromism triggered stable radical formation may pave a way for future
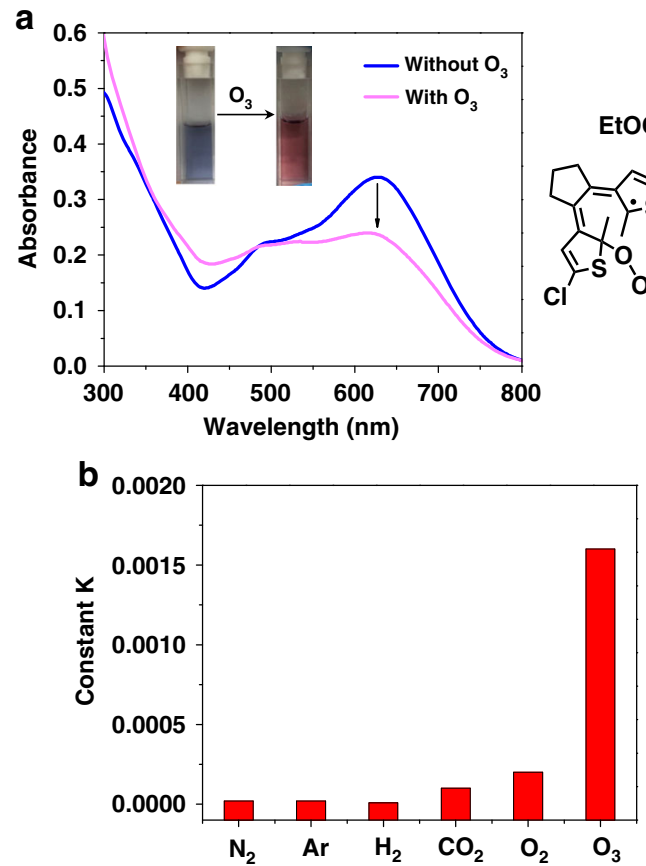

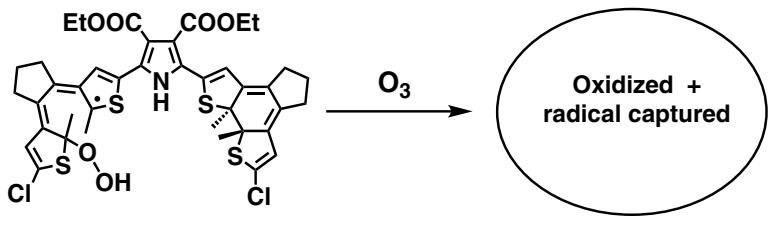

C

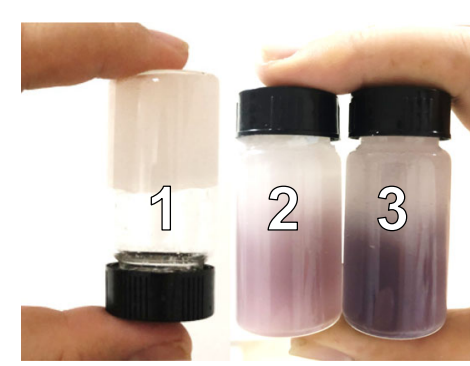

Fig. 5 Application for visualizing ozone detection. a UV-vis absorption spectral changes of compound 1 (1.0 $\times 10^{-5} \mathrm{M}$ ) in $\mathrm{CHCl}_{3}$ solution at the photostationary state in the presence of $\mathrm{O}_{3}$ at $25^{\circ} \mathrm{C}$. Inset: the corresponding solution color change. $\mathbf{b}$ The rate of absorbance changes at 630 nm of photoirradiated compound 1 in $\mathrm{CHCl}_{3}$ solution after bubbling with various types of gas. $\mathbf{c} \mathrm{A}$ gel of compound 1 containing chloroform: 1 . the original state; 2 . $\mathrm{O}_{3}$ bubbled photostationary state; 3 . the photostationary state. 
development of photochemical methodologies as well as the photomanipulation of advanced optoelectronic materials.

\section{Methods}

Chemicals and characterization. 1,2-bis(5-chloro-2-methyl-3-thienyl) cyclopentene $\mathrm{H}^{49}$ and compound $4^{50}$ were prepared and purified according to literature procedures, respectively. All other reagents were commercially available and used without further treatment. The synthesis details of the intermediates T1, T2, T3, T4, and T5 were shown in Supplementary Information. Thin-layer chromatography (TLC) analyses were performed on silica-gel plates, and flash chromatography was conducted by using silica-gel column packages purchased from Qingdao Haiyang Chemical Company, China. ${ }^{1} \mathrm{H}$ NMR and ${ }^{13} \mathrm{C}$ NMR spectra in $\mathrm{CDCl}_{3}$ or DMSO- $d_{6}$ were recorded on Brucker AM- 400 spectrometers with tetramethylsilane (TMS) as the internal standard. High-resolution mass spectrometry (HRMS) were recorded on a Waters LCT Premier XE spectrometer with methanol as solvent and a Matrix Assisted Laser Desorption Ionization-Time of Flight (MALDI-TOF) Mass Spectrometer (5800), respectively. UV-vis absorption spectra were recorded on a Shimadzu 1800 spectrophotometer, while the fluorescent emission spectra were taken with a Shimadzu RF-5301 PC; both spectrophotometers were standardized. HPLC were recorded on a Shimadzu LC-20A with Agilent C-18 column.

Synthesis of compound 1. Under nitrogen atmosphere, to a solution of compound $\mathrm{T} 1(0.370 \mathrm{~g}, 1.0 \mathrm{mmol})$ and freshly prepared T2 $(3.04 \mathrm{mmol})$ in degassed toluene $(20.0 \mathrm{~mL})$ was added $\mathrm{Pd}\left(\mathrm{PPh}_{3}\right)_{4}(0.173 \mathrm{~g}, 0.150 \mathrm{mmol})$ at room temperature, and the resulting mixture was purged with nitrogen for $30 \mathrm{~min}$ and then stirred overnight at $100{ }^{\circ} \mathrm{C}$. After cooling to room temperature, the mixture was concentrated and purified by column chromatography (silica gel, $\mathrm{CH}_{2} \mathrm{Cl}_{2} /$ petroleum ether $4: 1, \mathrm{v} / \mathrm{v})$ to give the compound $1(0.528 \mathrm{~g}, 66 \%)$ as a pale-yellow solid. ${ }^{1} \mathrm{H}$ NMR $\left(400 \mathrm{MHz}, \mathrm{CDCl}_{3}\right): \delta 1.32(\mathrm{t}, J=6.0 \mathrm{~Hz}, 6 \mathrm{H}), 1.87(\mathrm{~s}, 6 \mathrm{H}), 2.02-2.09(\mathrm{~m}$, $10 \mathrm{H}), 2.74(\mathrm{t}, J=8.0 \mathrm{~Hz}, 4 \mathrm{H}), 2.80(\mathrm{t}, J=8.0 \mathrm{~Hz}, 4 \mathrm{H}), 4.28(\mathrm{q}, J=8.0 \mathrm{~Hz}, 8 \mathrm{H}), 6.61$ (s, 2H), 7.00 (s, 2H), 8.09 (s, 1H). ${ }^{13} \mathrm{C}$ NMR $\left(100 \mathrm{MHz}, \mathrm{CDCl}_{3}\right): \delta 14.19,14.28$, $22.92,38.28,38.31,60.85,125.13,126.83,127.31,127.71,127.99,133.35,134.38$, 134.92, 135.05, 135.88, 136.37, 164.64. HRMS (ESI+): $[\mathrm{M}+\mathrm{Na}]^{+} \mathrm{calcd}$. for $\mathrm{C}_{40} \mathrm{H}_{39} \mathrm{NO}_{4} \mathrm{NaS}_{4} \mathrm{Cl}_{2}, \mathrm{~m} / \mathrm{z}$ : 818.1037; found, 818.1035.

Synthesis of compound 2. Under nitrogen atmosphere, to a solution of T3 ( $0.467 \mathrm{~g}$ $1.22 \mathrm{mmol})$ and freshly prepared T2 $(3.66 \mathrm{mmol})$ in degassed toluene $(25.0 \mathrm{~mL})$ was added $\mathrm{Pd}\left(\mathrm{PPh}_{3}\right)_{4}(0.212 \mathrm{~g}, 0.183 \mathrm{mmol})$ at room temperature, and the resulting mixture was purged with nitrogen for $30 \mathrm{~min}$ and then stirred overnight at $100{ }^{\circ} \mathrm{C}$. After cooling to room temperature, the mixture was concentrated and purified by column chromatography (silica gel, $\mathrm{CH}_{2} \mathrm{Cl}_{2} /$ petroleum ether $4: 1, \mathrm{v} / \mathrm{v}$ ) to give the compound $2(0.722 \mathrm{~g}, 71 \%)$ as a pale-yellow solid. ${ }^{1} \mathrm{H}$ NMR $\left(400 \mathrm{MHz}, \mathrm{CDCl}_{3}\right): \delta$ $1.21(\mathrm{t}, J=6.0 \mathrm{~Hz}, 6 \mathrm{H}), 1.91(\mathrm{~s}, 6 \mathrm{H}), 2.03-2.10(\mathrm{~m}, 10 \mathrm{H}), 2.73(\mathrm{t}, J=6.0 \mathrm{~Hz}, 4 \mathrm{H})$, $2.79(\mathrm{t}, J=6.0 \mathrm{~Hz}, 4 \mathrm{H}), 3.21(\mathrm{~s}, 3 \mathrm{H}), 4.18(\mathrm{q}, J=8.0 \mathrm{~Hz}, 4 \mathrm{H}), 6.58(\mathrm{~s}, 2 \mathrm{H}), 6.69(\mathrm{~s}$, $2 \mathrm{H}) .{ }^{13} \mathrm{C} \mathrm{NMR}\left(100 \mathrm{MHz}, \mathrm{CDCl}_{3}\right): \delta 14.11,22.96,32.74,38.12,38.19,60.51$, $116.19,125.08,126.05,126.91,129.30,131.34,133.20,134.25,135.07,135.18$, 135.50, 137.35, 164.44. HRMS (ESI+): $[\mathrm{M}+\mathrm{Na}]^{+}$calcd. for $\mathrm{C}_{41} \mathrm{H}_{41} \mathrm{NO}_{4} \mathrm{NaS}_{4} \mathrm{Cl}_{2}$, $\mathrm{m} / \mathrm{z}$ : 832.1193; found, 832.1172 .

Synthesis of compound 3. Under nitrogen atmosphere, to a mixture of T4 (0.145 g, $0.50 \mathrm{mmol})$, aqueous potassium carbonate $(10.0 \mathrm{~mL}, 1.7 \mathrm{M})$ and $\mathrm{Pd}\left(\mathrm{PPh}_{3}\right)_{4}$ $(0.058 \mathrm{~g}, 0.05 \mathrm{mmol})$ in degassed THF $(20.0 \mathrm{~mL})$ was added the solution of freshly prepared compound T5 $(0.75 \mathrm{mmol})$ using a syringe at room temperature, and the resulting mixture was purged with nitrogen for $30 \mathrm{~min}$ and then stirred overnight at reflux. After cooling to room temperature, the mixture was extracted with $\mathrm{CH}_{2} \mathrm{Cl}_{2}(20.0 \mathrm{~mL} \times 3)$, combined organic layers were washed with saturated $\mathrm{NaCl}$ solution $(20.0 \mathrm{~mL})$ and dried over anhydrous $\mathrm{Na}_{2} \mathrm{SO}_{4}$, filtrated, and concentrated. The residue was purified by column chromatography (silica gel, ethyl acetate/ petroleum ether $1: 3, \mathrm{v} / \mathrm{v})$ to give the compound $3(0.130 \mathrm{~g}, 49 \%)$ as a yellow solid. ${ }^{1} \mathrm{H} \mathrm{NMR}\left(400 \mathrm{MHz}, \mathrm{CDCl}_{3}\right): \delta 1.33(\mathrm{t}, J=6.0 \mathrm{~Hz}, 6 \mathrm{H}), 1.86(\mathrm{~s}, 3 \mathrm{H}), 1.97-2.05(\mathrm{~m}$, $5 \mathrm{H}), 2.71-2.78(\mathrm{~m}, 4 \mathrm{H}), 4.30(\mathrm{q}, J=8.0 \mathrm{~Hz}, 4 \mathrm{H}), 6.58(\mathrm{~s}, 1 \mathrm{H}), 6.96(\mathrm{~s}, 1 \mathrm{H}), 7.31(\mathrm{~s}$, $1 \mathrm{H}), 8.44(\mathrm{~s}, 1 \mathrm{H}) .{ }^{13} \mathrm{C}$ NMR $\left(100 \mathrm{MHz}, \mathrm{CDCl}_{3}\right): \delta 14.18,14.32,22.86,38.35,38.42$, $60.30,61.19,116.97,123.43,125.10,126.78,126.91,127.81,127.95,133.28,134.30$, 134.80, 134.95, 135.66, 136.00, 163.66, 165.72. HRMS (ESI+): $[\mathrm{M}+\mathrm{Na}]^{+}$calcd. for $\mathrm{C}_{25} \mathrm{H}_{26} \mathrm{NO}_{4} \mathrm{NaS}_{2} \mathrm{Cl}, \mathrm{m} / \mathrm{z}$ : 526.0903; found, 526.0898 .

Computational details. The structure of the compounds 1 and its closed forms were optimized at the DFT level of theory using the B3LYP51,52 functional and 6$31 \mathrm{G}(\mathrm{d})^{53}$ basis set. The structure of the open-shell structure $1^{\prime}$ was optimized by the same method but using the spin-unrestricted DFT formalism. The parameters of the g-tensor for the unpaired electron in the radical $1^{\prime}$ were calculated by gaugeindependent atomic orbital (GIAO) $\operatorname{method}^{54}$ using the B3LYP functional and 6$311++\mathrm{G}(\mathrm{d}, \mathrm{p})^{55-57}$ basis set. The solvent effect was accounted through the polarizable continuum model (PCM) ${ }^{58}$ by using $\mathrm{CHCl}_{3}$ as a model solvent $(\varepsilon=4.711)$. Using the ground state optimized geometries, the energies and intensities of singlet-singlet and doublet-doublet electronic transitions were estimated by TDDFT method ${ }^{59}$. The spin purity of doublet-doublet electronic transitions was concluded from the $\left\langle\mathrm{S}^{2}>\right.$ eigenvalues close to 0.75 . All the calculations have been carried out using the Gaussian 16 software ${ }^{60}$. The isosurfaces for molecular orbitals and spin densities were generated using the Chemissian software ${ }^{61}$.

\section{Data availability}

The data that support the findings of this study not included in the supplementary information document are available from the corresponding author upon reasonable request.

Received: 17 September 2019; Accepted: 30 January 2020; Published online: 18 February 2020

\section{References}

1. Zeng, Z. et al. Pro-aromatic and anti-aromatic $\pi$-conjugated molecules: an irresistible wish to be diradicals. Chem. Soc. Rev. 44, 6578-6596 (2015).

2. Geng, J. et al. Radical polymerization inside living cells. Nat. Chem. 11, 578-586 (2019).

3. Li, Z., Zou, X., Shi, F., Liu, R. \& Yagci, Y. Highly efficient dandelion-like nearinfrared light photoinitiator for free radical and thiol-ene photopolymerizations. Nat. Commun. 10, 3560 (2019).

4. Maxfield, M., Bloch, A. N. \& Cowan, D. O. Large electron acceptors for molecular metals: $13,13,14,14$-tetracyano-4,5,9,10-tetrahydro-2,7pyrenoquinodimethane (TCNTP) anions of 13,13,14,14-tetracyano-2,7pyrenoquinodimethane (TCNP). J. Org. Chem. 50, 1789-1796 (1985).

5. Casati, C., Franchi, P., Pievo, R., Mezzina, E. \& Lucarini, M. Unraveling unidirectional threading of $\alpha$-cyclodextrin in a [2] rotaxane through spin labeling approach. J. Am. Chem. Soc. 134, 19108-19117 (2012).

6. Rana, A. et al. Stable expanded porphycene-based diradicaloid and tetraradicaloid. Angew. Chem. Int. Ed. 57, 12534-12537 (2018).

7. Takahashi, T., Matsuoka, K.-I., Takimiya, K., Otsubo, T. \& Aso, Y. Extensive quinoidal oli-gothiophenes with dicyanomethylene groups at terminal positions as highly amphoteric redox molecules. J. Am. Chem. Soc. 127, 8928-8929 (2005)

8. Jiang, Q. et al. Diazuleno-s-indacene diradicaloids: syntheses, properties, and local (anti)aromaticity shift from neutral to dicationic state. Angew. Chem. Int. Ed. 57, 16737-16741 (2018).

9. Ooi, S., Shimizu, D., Furukawa, K., Tanaka, T. \& Osuka, A. Stable face-to-face singlet diradicaloids: triply linked corrole dimer gallium(III) complexes with two $\mu$-hydroxo-bridges. Angew. Chem. Int. Ed. 57, 14916-14920 (2018).

10. Zeng, Z. et al. Turning on the biradical state of tetracyano-perylene and quater-rylenequinodimethanes by incorporation of additional thiophene rings. Chem. Sci. 5, 3072-3080 (2014).

11. Ai., X. et al. Multispectral optoacoustic imaging of dynamic redox correlation and pathophysiological progression utilizing upconversion nanoprobes. Nat. Commun. 10, 1087 (2019).

12. Zhu, C. et al. Extraordinary redox activities in ladder-type conjugated molecules enabled by $\mathrm{B} \leftarrow \mathrm{N}$ coordination-promoted delocalization and hyperconjugation. J. Am. Chem. Soc. 140, 18173-18182 (2018).

13. Zhu, L. et al. Unimolecular photoconversion of multicolor luminescence on hierarchical self-assemblies. J. Am. Chem. Soc. 135, 5175-5182 (2013).

14. Kim, J.-H., Lee, E., Jeong, Y.-H. \& Jang, W.-D. Unique photoluminescence of diacetylene containing dendrimer self-assemblies: application in positive and negative luminescence patterning. Chem. Mater. 24, 2356-2363 (2012).

15. Zhou, H. et al. Photoswitching of glass transition temperatures of azobenzenecontaining polymers induces reversible solid-to-liquid transitions. Nat. Chem. 9, 145-151 (2017)

16. Zhu, L. et al. Engineering topochemical polymerizations using block copolymer templates. J. Am. Chem. Soc. 136, 13381-13387 (2014).

17. Zhou, Y. \& Zhu, L. Involving synergy of green light and acidic responses in control of unimolecular multicolor luminescence. Chem. Eur. J. 24, 10306-10309 (2018)

18. Irie, M. Diarylethenes for memories and switches. Chem. Rev. 100, 1685-1716 (2000).

19. Zhang, J., Zou, Q. \& Tian, H. Photochromic materials: more than meets the eye. Adv. Mater. 25, 378-399 (2013).

20. Myles, A. J., Zhang, Z., Liu, G. \& Branda, N. R. Novel synthesis of photochromic polymers via ROMP. Org. Lett. 2, 2749-2751 (2000).

21. Nakatani, K. \& Yu, P. Photochromic magnetic materials. Adv. Mater. 13, 1411-1413 (2001)

22. Zhou, Y., Zou, Q., Qiu, J., Wang, L. \& Zhu, L. Rational design of a green-lightmediated unimolecular platform for fast switchable acidic sensing. J. Phys. Chem. Lett. 9, 550-556 (2018).

23. Wu, N. M.-W., Ng, M., Lam, W. H., Wong, H.-L. \& Yam, V. W.-W. Photochromic heterocycle-fused thieno[3,2-b]phosphole oxides as visible light 
switches without sacrificing photoswitching efficiency. J. Am. Chem. Soc. 139, 15142-15150 (2017).

24. Li, M. et al. Light-driven chiral switching of supramolecular metallacycles with photoreversibility. Chem 5, 1-15 (2019).

25. Pu, S., Sun, Q., Fan, C., Wang, R. \& Liu, G. Recent advances in diarylethene-based multi-responsive molecular switches. J. Mater. Chem. C. 4, 3075-3093 (2016).

26. Matsuda, K., Matsuo, M., Mizoguti, S., Higashiguchi, K. \& Irie, M. Reversed photoswitching of intramolecular magnetic interaction using a photochromic bis(2-thienyl)ethene spin coupler. J. Phys. Chem. B 106, 11218-11225 (2002).

27. Chen, Y., Pang, M., Cheng, K., Wang, Y. \& Meng, J. Synthesis and properties of novel photochromic biindenylidenedione derivative bearing TEMPO radical. J. Photoch. Photobio. A 194, 122-128 (2008).

28. Chen, Y., Pang, M., Cheng, K., Wang, Y. \& Meng, J. Molecular system based on novel photochromic biindenylidenedione derivative demonstrating photomodulation of magnetism. Chin. J. Chem. 30, 1759-1765 (2012).

29. Chen, Y. \& Meng, J. Synthesis and properties of novel photomodulation magnetic molecular-based magnet based on photochromic biindenylidenedione derivative. Chin. J. Org. Chem. 36, 1869-1877 (2016).

30. Hayashi, T. \& Maeda, K. Preparation of a new phototropic substance. Bull. Chem. Soc. Jpn. 33, 565-566 (1960).

31. Hatano, S. \& Abe, J. A peroxide-bridged imidazole dimer formed from a photochromic naphthalene-bridged imidazole dimer. Phys. Chem. Chem. Phys. 14, 5855-5860 (2012).

32. Kishimoto, Y. \& Abe, J. A fast photochromic molecule that colors only under UV light. J. Am. Chem. Soc. 131, 4227-4229 (2009).

33. Wang, S. et al. $\mathrm{Cu}^{2+}$-selectivity gated photochromism in Schiff-modified diarylethenes with a star-shaped structure. J. Mater. Chem. C. 5, 282-289 (2017).

34. Cai, Y. et al. Enabling light work in helical self-assembly for dynamic amplification of chirality with photoreversibility. J. Am. Chem. Soc. 138, 2219-2224 (2016).

35. Nishinaga, T. et al. Biradical character of linear $\pi$-conjugated oligomer dications composed of thiophene, pyrrole, and methylthio end-capping units. Org. Lett. 12, 5374-5377 (2010).

36. Hicks, R. G. What's new in stable radical chemistry? Org. Biomol. Chem. 5 , 1321-1338 (2007).

37. Zhu, L. et al. Luminescent color conversion on cyanostilbene-functionalized quantum dots via in-situ photo-tuning. Adv. Mater. 24, 4020-4024 (2012).

38. Zhang, J., Wang, J. \& Tian, H. Taking orders from light: progress in photochromic bio-materials. Mater. Horiz. 1, 169-184 (2014).

39. Pell, A. J., Pintacuda, G. \& Grey, C. P. Paramagnetic NMR in solution and the solid state. Prog. Nucl. Mag. Res. Sp. 111, 1-271 (2019).

40. Liu, K., Ryan, D., Nakanishi, K. \& McDermott, A. Solid State NMR studies of paramagnetic coordination complexes: a comparison of protons and deuterons in detection and decoupling. J. Am. Chem. Soc. 117, 6897-6906 (1995).

41. Li, R. et al. Achieving overall water splitting using titanium dioxide-based photocatalysts of different phases. Energy Environ. Sci. 8, 2377-2382 (2015).

42. Semeluk, G. P. \& Unger, I. Benzene photosensitized decomposition of chloroform. Nature 198, 853-855 (1963).

43. Dong, X., Hu, F., Liu, Z., Zhang, G. \& Zhang, D. A fluorescent turn-on low dose detection of gamma-radiation based on aggregation-induced emission. Chem. Commun. 51, 3892-3895 (2015).

44. Schreckenbach, G. \& Ziegler, T. Calculation of the g-tensor of electron paramagnetic resonance spectroscopy using gauge-including atomic orbitals and density functional theory. J. Phys. Chem. A 101, 3388-3399 (1997).

45. Stoll, S. et al. Structure of the biliverdin radical intermediate in phycocyanobilin: ferredoxin oxidoreductase identified by high-field EPR and DFT. J. Am. Chem. Soc. 131, 1986-1995 (2009).

46. Mansoorabadi, S. O. et al. EPR spectroscopic and computational characterization of the hydroxyethylidene-thiamine pyrophosphate radical intermediate of pyruvate: ferredoxin oxidoreductase. Biochem 45, 7122-7131 (2006).

47. Mattson, M. P. Pathways towards and away from Alzheime's disease. Nature 430, 631-639 (2004)

48. Yamazoe, N. \& Miura, N. Environmental gas sensing. Sens. Actuators B 20, 95-102 (1994)

49. Zou, Q., Jia, J., Xu, B., Ding, L. \& Tian, H. New photochromic chemosensors for $\mathrm{Hg}^{2+}$ and $\mathrm{F}^{-}$. Tetrahedron 67, 915-921 (2011).

50. Algi, M. P., Öztaş, Z. \& Algi, F. Triple channel responsive $\mathrm{Cu}^{2+}$ probe. Chem. Commun. 48, 10219-10221 (2012).

51. Becke, A. D. Density-functional thermochemistry. III. The role of exact exchange. J. Chem. Phys. 98, 5648-5652 (1993).

52. Lee, C., Yang, W. \& Parr, R. G. Development of the Colle-Salvetti correlationenergy formula into a functional of the electron density. Phys. Rev. B 37, 785-789 (1988).

53. Hehre, W. J., Ditchfield, R. \& Pople, J. A. Self-consistent molecular orbital methods. XII. Further extensions of Gaussian-type basis sets for use in molecular orbital studies of organic molecules. J. Chem. Phys. 56, 2257-2261 (1972).
54. Wolinski, K., Hilton, J. F. \& Pulay, P. Efficient implementation of the gaugeindependent atomic orbital method for NMR chemical shift calculations. J. Am. Chem. Soc. 112, 8251-8260 (1990).

55. Raghavachari, K. et al. Self-consistent molecular orbital methods. XX. Basis set for correlated wave-functions. J. Chem. Phys. 72, 650-654 (1980).

56. Clark, T., Chandrasekhar, J., Spitznagel, W. \& Schleyer, P. V. R. Efficient diffuse function-augmented basis-sets for anion calculations. III. The 3-21+G basis set for first-row elements, Li-F. J. Comp. Chem. 4, 294-301 (1983).

57. Frisch, M. J., Pople, J. A. \& Binkley, J. S. Self-consistent molecular orbital methods. 25. Supplementary functions for Gaussian basis sets. J. Chem. Phys. 80, 3265-3269 (1984)

58. Tomasi, J., Mennucci, B. \& Cammi, R. Quantum mechanical continuum solvation models. Chem. Rev. 105, 2999-3093 (2005).

59. Casida, M. E., Jamorski, C., Casida, K. C. \& Salahub, D. R. Molecular excitation energies to high-lying bound states from time-dependent densityfunctional response theory: characterization and correction of the timedependent local density approximation ionization threshold. J. Chem. Phys. 108, 4439-4449 (1998).

60. Frisch, M. J. et al. Gaussian 16, Revision A.03.(Gaussian, Inc, Wallingford, 2016)

61. Skripnikov, L. Chemissian V. 4.53, http://www.chemissian.com/.

\section{Acknowledgements}

This work was supported by the Natural Science Foundation of Shanghai (17ZR1447100) and NSFC (21406137), and partially by the National Key Research and Development Program of China (Grant 2017YFA0207700) and the Science and Technology Commission of Shanghai Municipality (19DZ2271100). The theoretical calculations were performed with computational resources provided by the High Performance Computing Center North (HPC2N) which is a Swedish national center for Scientific and Parallel Computing through the project "Multiphysics Modeling of Molecular Materials" SNIC 2017-12-49. G.B. and H.Å. thank the support of Carl Tryggers foundation (Grant No. CTS 17:537), Olle Engkvist Byggmästare foundation (contract No. 189-0223). We thank Dr. Yunyao Xu, Dr. Brandon Fowler and Dr. Xiao Xiao from Columbia University for assistance with the NMR and MS analysis.

\section{Author contributions}

L.Z., Q.Z., and X.C. conceived this project and designed the experiments. X.C., W.Z., and Q.Z. did the synthetic work. X.C., W.Z., J.G., and Y.Z. carried out the photophysical studies as well as the structural characterization. G.B. and H.Å. carried out the calculation studies. M.L.S., Q.Z., W.C., and L.Z. contributed to data and mechanism analysis. X.C., Q.Z., and L.Z. wrote the paper and the other authors helped revising the paper.

\section{Competing interests}

The authors declare no competing interests.

\section{Additional information}

Supplementary information is available for this paper at https://doi.org/10.1038/s41467 020-14798-9.

Correspondence and requests for materials should be addressed to Q.Z., W.C. or L.Z.

Peer review information Nature Communications thanks the anonymous reviewers for their contribution to the peer review of this work.

Reprints and permission information is available at http://www.nature.com/reprints

Publisher's note Springer Nature remains neutral with regard to jurisdictional claims in published maps and institutional affiliations.

pen Access This article is licensed under a Creative Commons Attribution 4.0 International License, which permits use, sharing, adaptation, distribution and reproduction in any medium or format, as long as you give appropriate credit to the original author(s) and the source, provide a link to the Creative Commons license, and indicate if changes were made. The images or other third party material in this article are included in the article's Creative Commons license, unless indicated otherwise in a credit line to the material. If material is not included in the article's Creative Commons license and your intended use is not permitted by statutory regulation or exceeds the permitted use, you will need to obtain permission directly from the copyright holder. To view a copy of this license, visit http://creativecommons.org/ licenses/by/4.0/

(C) The Author(s) 2020 